\title{
Power spectrum of stochastic wave and diffusion equations in the warm inflation models
}

\author{
Z. Haba $^{1, \mathrm{a}}$ (1) \\ ${ }^{1}$ Institute of Theoretical Physics, University of Wroclaw, 50-204 Wroclaw, Poland
}

Received: 22 April 2020 / Accepted: 10 June 2020 / Published online: 22 June 2020

(C) The Author(s) 2020

\begin{abstract}
We discuss dissipative stochastic wave and diffusion equations resulting from an interaction of the inflaton with an environment in an external expanding homogeneous metric. We show that a diffusion equation well approximates the wave equation in a strong friction limit. We calculate the long wave power spectrum of the wave equation under the assumption that the perturbations are slowly varying in time and the expansion is almost exponential. Under the assumption that the noise has a form invariant under the coordinate transformations we obtain the power spectrum close to the scale invariant one. In the diffusion approximation we go beyond the slow variation assumption. We calculate the power spectrum exactly in models with exponential inflation and polynomial potentials and with power-law inflation and exponential potentials.
\end{abstract}

\section{Introduction}

It has been discovered long time ago by Harrison [1] and Zeldovich [2] that the scale invariant spectrum of galaxy distribution plays an essential role in the galaxy formation after the Big Bang. The spectrum close to the scale invariant one has been confirmed by WMAP observations [3,4]. Since then the power spectrum is a substantial criterion for a validity of cosmological models. The power spectrum results from quantization of the quadratic fluctuations around the homogeneous solution [5-11]. The formalism is explicitly gauge invariant $[12,13]$. It treats quantum gravitational fluctuations and inflaton fluctuations on the same footing. Nevertheless, it has been shown [14-17] that if the quantum inflaton fluctuations are expressed by stochastic fluctuations in e-fold time then the inflaton fluctuations already contain the gravitational fluctuations leading to the same power spectrum as in [13]. The scalar field models can be considered as effective field theories of scalar cosmological perturba-

a e-mail: zbigniew.haba@uwr.edu.pl (corresponding author) tions. The power spectrum close to the scale invariant one has also been obtained in warm inflation models $[18,19]$. The model of warm inflation is treated as an effective field theory of an inflaton interacting with a large number of other fields $[20,21]$. The inflaton wave equation becomes stochastic as a result of an interaction with other fields whose effect is described on the the basis of their contribution to the entropy and to the energy density $[18,22,23]$. In this paper we study in detail the stochastic wave equation in the form derived from an interaction of scalar fields with an environment [2426]. In this model the heat bath is an initial state of an infinite set of scalar massive fields $\chi_{n}$ interacting with the inflaton. The fields $\chi_{n}$ are treated as unobservable degrees of freedom. We average over these degrees of freedom. As a result of the interaction with $\chi_{n}$ the inflaton acquires a friction and a noise term. The stochastic model is considered as a Markovian approximation to the Hamiltonian model of the $\chi_{n}$ fields interacting with the inflaton. Such an approach is analogous to the treatment of a Brownian particle in an environment of other particles.

In this paper we first repeat a calculation of the power spectrum in an extended model of the inflaton-environment interaction [26] on the basis of previously developed methods $[27,28]$ under the assumption that the perturbations of the non-linear wave equation are slowly varying in time (are almost constant). However, the main objective here is a development of another tool for a computation of the power spectrum based on the approximation of the dissipative wave equation by a diffusion equation. In the case of a random diffusion the calculation of the power spectrum is much simpler. Its dependence on the evolution law can be seen in a more transparent way. We are able to calculate the power spectrum with parameters which can substantially vary in time.

The plan of the paper is the following. In Sect. 2 we repeat the main steps of the derivation [26] of the model emphasizing the extra terms which appear in comparison to the warm 
inflation inflaton equation [21]. We discuss the resulting dissipative stochastic wave equation for an inflaton interacting with an environment by means of a potential $U$. In Sect. 3 we show that at strong friction the solutions of the wave equation tend to the solutions of a diffusion equation. In Sect. 4 we calculate the power spectrum of the stochastic wave equation under the assumption that the evolution of the scale factor is almost exponential and the variables in this equation can be treated as constants. This is a repetition of the standard calculations $[19,27,28]$ but in a model with different potentials and a different noise. In Sect. 5 we calculate the power spectrum of the stochastic diffusion equation assuming again that the expansion of the metric is almost exponential and that the variables in this equation can be treated as constants. We obtain the same power spectrum as in the case of the wave equation,i.e., an almost scale invariant spectrum, which is shown to be a consequence of the form of the noise. We discuss a relation of our results to the ones in the literature. In Sect. 6 we study solutions of the diffusion equation with almost exponential expansion but with varying potentials. We obtain a shift in the formula for the spectral index. Then, we explore exponential potentials in a power-law expanding metric when the method of constant parameters does not apply. The diffusion equation reveals a sensitive dependence of the power spectrum on the potentials. In Appendix A we show in a simple way that the form of the noise that leads to the scale invariant spectrum follows from its invariance under coordinate transformations. In Appendix B we give a simplified derivation of the scale invariant spectrum showing the crucial role of the form of the noise and the exponential expansion.

\section{The model of an environment}

We recall the basic ingredients of the model [24-26] of an interaction of the inflaton with an environment. We consider the Lagrangian

$$
\begin{aligned}
\mathcal{L}= & \frac{1}{2} \partial_{\mu} \phi \partial^{\mu} \phi-V(\phi) \\
& +\sum_{n}\left(\frac{1}{2} \partial_{\mu} \chi_{n} \partial^{\mu} \chi_{n}-\frac{1}{2} m_{n}^{2} \chi_{n} \chi_{n}-\lambda_{n} U(\phi) \chi_{n}\right),
\end{aligned}
$$

where $U(\phi)$ is a certain interaction potential. Equations of motion read

$$
\begin{aligned}
& g^{-\frac{1}{2}} \partial_{\mu}\left(g^{\frac{1}{2}} \partial^{\mu} \phi\right)=-V^{\prime}-U^{\prime}(\phi) \sum_{n} \lambda_{n} \chi_{n}, \\
& g^{-\frac{1}{2}} \partial_{\mu}\left(g^{\frac{1}{2}} \partial^{\mu} \chi_{n}\right)+m_{n}^{2} \chi_{n}=-\lambda_{n} U(\phi),
\end{aligned}
$$

where $g_{\mu \nu}$ is the metric tensor and $g=\left|\operatorname{det}\left[g_{\mu \nu}\right]\right|$. We restrict ourselves to the flat expanding metric

$d s^{2}=g_{\mu \nu} d x^{\mu} d x^{\nu}=d t^{2}-a^{2} d \mathbf{x}^{2}$

We write

$\chi_{n}=a^{-\frac{3}{2}} \tilde{\chi}_{n}$.

Then, in the momentum space Eq. (3) reads

$\partial_{t}^{2} \tilde{\chi}_{n}+\omega_{n}^{2} \tilde{\chi}_{n}=-\lambda_{n} a^{\frac{3}{2}} U(\phi)$,

where

$\omega_{n}^{2}=a^{-2} k^{2}+m_{n}^{2}-\frac{3}{2} \partial_{t} H-\frac{9}{4} H^{2}$

$H=a^{-1} \partial_{t} a$. Let us consider low momenta $a^{-1} k<<H$ so that for a large time we can neglect $a^{-2} k^{2}$ term. We also assume that $H$ is slowly varying and $\omega_{n}^{2}>0$.

We can solve Eq. (3) for $\chi_{n}$

$\chi_{n}=\chi_{n}^{c l}-\lambda_{n} \int d x^{\prime} G_{n}\left(x, x^{\prime}\right) U\left(x^{\prime}\right)$,

where we denote $U(x)=U(\phi(x)), \chi_{n}^{c l}$ are solutions of the linear equation and $G_{n}$ is the Green function. When we insert $\chi_{n}$ of Eq. (7) in Eq. (2) then it takes the form

$g^{-\frac{1}{2}} \partial_{\mu}\left(g^{\frac{1}{2}} \partial^{\mu} \phi\right)+V^{\prime}=-U^{\prime} \sum_{n} \lambda_{n}^{2} G_{n} U+U^{\prime} \eta \equiv \delta \phi+U^{\prime} \tilde{\eta}$,

where

$\tilde{\eta}=-\sum_{n} \lambda_{n} \chi_{n}^{c l}$

When we calculate the expectation value of $\tilde{\eta}$ over the free field solutions $\chi_{n}^{c l}$ with respect to the Gibbs measure (with temperature $\beta^{-1}$ ) then approximately

$$
\begin{gathered}
\left\langle\tilde{\eta}(x) \tilde{\eta}\left(x^{\prime}\right)\right\rangle=\beta^{-1}(2 \pi)^{-3} \int d \mathbf{k} \exp \left(i \mathbf{k}\left(\mathbf{x}-\mathbf{x}^{\prime}\right)\right) \\
\sum_{n} \lambda_{n}^{2} \omega_{n}^{-2} \cos \left(\omega_{n}\left(t-t^{\prime}\right)\right) .
\end{gathered}
$$

We assume that $\lambda_{n}^{2} \omega_{n}^{-2} \simeq \gamma^{2}$ is a constant. Then

$$
\left\langle\tilde{\eta}(x) \tilde{\eta}\left(x^{\prime}\right)\right\rangle=\beta^{-1} \gamma^{2} a^{-3} \delta\left(\mathbf{x}-\mathbf{x}^{\prime}\right) \delta\left(t-t^{\prime}\right) .
$$

The friction $\delta \phi$ in Eq. (8) can be expressed by means of an approximate Green function

$$
\frac{\sin \left(\omega_{n}(t-s)\right)}{\omega_{n}}=\partial_{s}\left(\frac{\cos \left(\omega_{n}(t-s)\right)}{\omega_{n}^{2}}\right)
$$


(in Eq.(7) $x=(t, \mathbf{x})$ and $\left.x^{\prime}=\left(s, \mathbf{x}^{\prime}\right)\right)$. After an integration by parts in $G_{n} U$ the friction term takes the form

$$
-a(t)^{-\frac{3}{2}} U^{\prime} \sum_{n} \lambda_{n}^{2} \int \omega_{n}^{-2} \cos \left(\omega_{n}(t-s)\right) \partial_{s}\left(a(s)^{\frac{3}{2}} U\right) d s .
$$

We have got the same kernel as the one in Eq. (10) which we approximated by $\delta(t-s)$ in Eq. (11) for the correlation of the noise. With these approximations, when we perform the differentiation in Eq. (12), then Eq. (2)in the flat expanding metric (4) reads (we change the notation $\phi \rightarrow \phi_{\eta}$ )

$$
\begin{aligned}
& \partial_{t}^{2} \phi_{\eta}-a^{-2} \Delta \phi_{\eta}+\left(3 H+\gamma^{2}\left(U^{\prime}\right)^{2}\right) \partial_{t} \phi_{\eta} \\
& \quad+V^{\prime}\left(\phi_{\eta}\right)+\frac{3}{2} \gamma^{2} H U U^{\prime}\left(\phi_{\eta}\right)=\beta^{-\frac{1}{2}} \gamma a^{-\frac{3}{2}} U^{\prime} \eta
\end{aligned}
$$

where we wrote $\tilde{\eta}=\gamma \beta^{-\frac{1}{2}} a^{-\frac{3}{2}} \eta$ so that

$$
\left\langle\eta_{s}(\mathbf{x}) \eta_{t}(\mathbf{y})\right\rangle=\delta(t-s) \delta(\mathbf{x}-\mathbf{y}) .
$$

We consider a linearized form of Eq. (13) resulting from an expansion around its homogeneous (space-independent) solution

$\partial_{t}^{2} \phi_{c}+\left(3 H+\gamma^{2} U^{\prime}\left(\phi_{c}\right)^{2}\right) \partial_{t} \phi_{c}+V^{\prime}\left(\phi_{c}\right)+\frac{3}{2} \gamma^{2} H U U^{\prime}\left(\phi_{c}\right)=0$

We write $\phi_{\eta}=\phi_{c}+\phi$. The initial conditions are contained in $\phi_{c}$, so we assume zero as the intitial condition for $\phi$. The linearization of Eq. (13) expanded about $\phi_{c}$ reads

$$
\begin{aligned}
\partial_{t}^{2} \phi & -a^{-2} \Delta \phi+\left(3 H+\gamma^{2} U^{\prime}\left(\phi_{c}\right)^{2}\right) \partial_{t} \phi+V^{\prime \prime}\left(\phi_{c}\right) \phi \\
& +2 \gamma^{2} U^{\prime} U^{\prime \prime}\left(\phi_{c}\right) \partial_{t} \phi_{c} \phi+\frac{3}{2} \gamma^{2} H\left(\left(U^{\prime}\right)^{2}+U U^{\prime \prime}\right) \phi \\
= & \beta^{-\frac{1}{2}} \gamma a^{-\frac{3}{2}} U^{\prime}\left(\phi_{c}\right) \eta .
\end{aligned}
$$

We can transform Eq. (16) to another form . Let

$$
\phi=a^{-\frac{3}{2}} \exp \left(-\frac{1}{2} \gamma^{2} \int_{0}^{t} U^{\prime}\left(\phi_{c}\right)^{2}\right) \Phi
$$

Then

$$
\begin{aligned}
\partial_{t}^{2} & \Phi-a^{-2} \Delta \Phi-\Omega^{2} \Phi \\
& =\beta^{-\frac{1}{2}} \gamma \exp \left(\frac{1}{2} \gamma^{2} \int_{0}^{t} U^{\prime}\left(\phi_{c}\right)^{2}\right) U^{\prime}\left(\phi_{c}\right) \eta_{t},
\end{aligned}
$$

where

$$
\begin{aligned}
\Omega^{2}=- & V^{\prime \prime}-\frac{1}{2} \gamma^{2} \partial_{t}\left(U^{\prime}\right)^{2}+\frac{3}{2} \partial_{t} H \\
& -\frac{3}{2} \gamma^{2} H\left(\left(U^{\prime}\right)^{2}+U U^{\prime \prime}\right)+\frac{1}{4}\left(3 H+\gamma^{2}\left(U^{\prime}\right)^{2}\right)^{2} .
\end{aligned}
$$

The wave equation with friction is transformed into a wave equation with a complex mass $\Omega$. Note that large $3 H+$ $\gamma^{2}\left(U^{\prime}\right)^{2}$ means large $\Omega$.

\section{Diffusion approximation}

In this section we show that the diffusion approximation to Eq. (16), i.e., the omission of $\partial_{t}^{2} \phi$, is equivalent to the neglect of fast decaying modes (for a large $\Omega$ ) in the solution of Eqs. (17)-(18). The diffusion approximation to Eq. (16) in the momentum space reads (we denote the Fourier transform $\tilde{\phi}(\mathbf{k})$ by the same letter as its spatial form $\phi(\mathbf{x}))$

$$
\begin{aligned}
& \left(3 H+\gamma^{2} U^{\prime}\left(\phi_{c}\right)^{2}\right) \partial_{t} \phi+a^{-2} k^{2} \phi++V^{\prime \prime}\left(\phi_{c}\right) \phi \\
& \quad+2 \gamma^{2} U^{\prime} U^{\prime \prime}\left(\phi_{c}\right) \partial_{t} \phi_{c} \phi \\
& \quad+\frac{3}{2} \gamma^{2} H\left(\left(U^{\prime}\right)^{2}+U U^{\prime \prime}\right) \phi=\beta^{-\frac{1}{2}} \gamma a^{-\frac{3}{2}} U^{\prime}\left(\phi_{c}\right) \eta .
\end{aligned}
$$

On the other hand we may express the solution of Eq. (18) (momentum space) with zero initial condition at $t_{0}$ by means of the Green function $G$

$\Phi(t)=\beta^{-\frac{1}{2}} \gamma \int_{t_{0}}^{t} G(t, s) \exp \left(\frac{1}{2} \gamma^{2} \int_{0}^{s} U^{\prime}\left(\phi_{c}\right)^{2}\right) U^{\prime}\left(\phi_{c}\right) \eta_{s} d s$,

where the approximate Green function (for large slowly varying $\omega$ ) is

$G(t, s)=\omega(s)^{-\frac{1}{2}} \omega(t)^{-\frac{1}{2}} \sinh \left(\int_{s}^{t} d \tau \omega(\tau)\right)$

with

$\omega^{2}=\Omega^{2}-a^{-2} \mathbf{k}^{2}$.

Expanding $\omega$ in powers of $\left(3 H+\tilde{\gamma}^{2}\right)^{-1}$ where

$\tilde{\gamma}^{2}=\gamma^{2}\left(U^{\prime}\right)^{2}$ 
we obtain in the lowest order of the expansion

$$
\begin{aligned}
\omega= & \frac{3}{2} H+\frac{1}{2} \tilde{\gamma}^{2}+\left(3 H+\tilde{\gamma}^{2}\right)^{-1}\left(-a^{-2} k^{2}-V^{\prime \prime}\right. \\
& -2 \gamma^{2} U^{\prime} U^{\prime \prime} \partial_{t} \phi_{c} \\
& \left.-\frac{3}{2} \gamma^{2} H\left(\left(U^{\prime}\right)^{2}+U U^{\prime \prime}\right)+\frac{3}{2} \partial_{t} H+\frac{1}{2} \gamma^{2} \partial_{t}\left(U^{\prime}\right)^{2}\right) \\
\equiv & \frac{3}{2} H+\frac{1}{2} \tilde{\gamma}^{2}+\frac{1}{2} \partial_{t} \ln \left(3 H+\tilde{\gamma}^{2}\right)-v,
\end{aligned}
$$

where

$$
\begin{aligned}
v= & \left(3 H+\tilde{\gamma}^{2}\right)^{-1}\left(a^{-2} k^{2}+V^{\prime \prime}+\frac{1}{2} \partial_{t} \tilde{\gamma}^{2}\right. \\
& \left.+\frac{3}{2} \gamma^{2} H\left(\left(U^{\prime}\right)^{2}+U U^{\prime \prime}\right)\right) .
\end{aligned}
$$

We compare solutions of the wave equation (16) with solutions of the diffusion equation (19). The solution of the diffusion equation (19) is

$$
\begin{gathered}
\phi_{t}=\beta^{-\frac{1}{2}} \gamma \int_{t_{0}}^{t} \exp \left(-\int_{s}^{t} d \tau v(\tau)\right) \\
\left(3 H(s)+\tilde{\gamma}^{2}\right)^{-1} a(s)^{-\frac{3}{2}} U^{\prime}\left(\phi_{c}\right) \eta(s) d s .
\end{gathered}
$$

We compare the solution (26) with $(20,21)$. In the Green function (21) we have

$$
\begin{aligned}
& \int_{s}^{t} d \tau \omega(\tau)=\frac{1}{2} \int_{s}^{t}\left(3 H(\tau)+\tilde{\gamma}(\tau)^{2}\right) d \tau \\
& \quad+\frac{1}{2} \ln \left(3 H(t)+\tilde{\gamma}(t)^{2}\right)-\frac{1}{2} \ln \left(3 H(s)+\tilde{\gamma}(s)^{2}\right) \\
& \quad-\int_{s}^{t} d \tau v(\tau) .
\end{aligned}
$$

If in

$\sinh (X)=\frac{1}{2} \exp (X)-\frac{1}{2} \exp (-X)$

we neglect the second term as quickly vanishing (for $X>0$ ) and in Eq. (21) $\omega(s)^{-\frac{1}{2}}$ is approximated by $\left(\frac{3}{2} H+\frac{1}{2} \tilde{\gamma}^{2}\right)^{-\frac{1}{2}}$ (and the same approximation for $\omega(t)^{-\frac{1}{2}}$ ) then a simple comparison of Eqs. $(20,21)$ and $(26,27)$ leads to the conclusion that for large $3 H+\tilde{\gamma}^{2}$ the solutions of the wave equation and the diffusion equation (with zero initial conditions) coincide.

\section{Power spectrum of the linearized wave equation}

We have calculated the power spectrum in the EinsteinKlein-Gordon system in [28] in the case $U(\phi)=\phi$. The changes corresponding to the replacement $\phi \rightarrow U(\phi)$ are the following: $3 H+\gamma^{2} \rightarrow 3 H+\tilde{\gamma}^{2}, V^{\prime \prime} \rightarrow V^{\prime \prime}+$ $\frac{1}{2} \partial_{t} \tilde{\gamma}^{2}, \frac{3}{2} \sigma \gamma^{2} H \rightarrow \frac{3}{2} \gamma^{2} H\left(\left(U^{\prime}\right)^{2}+U U^{\prime \prime}\right)$. We repeat here the main steps of [28] in order to fix the stage for the discussion of the extended model. We still rewrite the correspondence in a different way. Let

$\tilde{\Gamma}=(3 H)^{-1} \tilde{\gamma}^{2}$

replacing $\Gamma$ from [28],

$Q=\frac{1}{2} \partial_{t} \tilde{\gamma}^{2}+\tilde{\gamma}^{2}\left(U^{\prime}\right)^{-2} U U^{\prime \prime}$

and

$\delta=\left(V^{\prime \prime}+Q\right)\left(3 H^{2}\right)^{-1}$

replacing $\eta$ from [28]. If in Eq. (15) we applied the slow roll approximation then we could express $\partial_{t} \phi_{c}$ in $Q$ by derivatives of the potential $U$.

The power spectrum $\rho$ of fluctuations $\phi$ is defined by

$\left\langle\phi_{t}(\mathbf{x}) \phi_{t}(\mathbf{y})\right\rangle=\int d \mathbf{k} \rho_{t}(\mathbf{k}) \exp (i \mathbf{k}(\mathbf{x}-\mathbf{y}))$

or in Fourier transform

$\left\langle\phi_{t}(\mathbf{k}) \phi_{t}\left(\mathbf{k}^{\prime}\right)\right\rangle=(2 \pi)^{3} \delta\left(\mathbf{k}+\mathbf{k}^{\prime}\right) \rho_{t}(\mathbf{k})$.

The spectral index $2 \kappa$ is defined by the low $k=|\mathbf{k}|$ behaviour $\rho_{t}(\mathbf{k}) \simeq k^{-2 \kappa}$.

In [28] we have calculated the spectrum under the assumption that $\delta$ and $\tilde{\Gamma}$ are almost constant. If $H$ is varying in time then estimates by means of the methods [28] (based on [27]) are not reliable for varying parameters. For this reason in the next section we discuss the diffusion approximation when the time evolution can be treated in a more controllable way. We define

$\epsilon=-H^{-2} \partial_{t} H$.

Without the thermal noise $(\gamma=0) \epsilon$ can be expressed from Friedmann equations as $\frac{1}{8 \pi G}\left(V^{\prime}\right)^{2} V^{-2}$ (where $G$ is the Newton constant). With the thermal noise and the interaction $U$ the formula for $\epsilon$ in terms of potentials is more involved (see [26],eq. (89)). We keep (33) as a definition of $\epsilon$ and do not attempt to express it by potentials.

We introduce the conformal time

$\tau=\int d t a^{-1}$.

With a slowly varying $H$ we have approximately

$a H=-(1-\epsilon)^{-1} \frac{1}{\tau}$. 
Equation (35) can be obtained by an integration of the identity [29]

$\partial_{t}((1-\epsilon) H a)^{-1}=-a^{-1}+\partial_{t} \epsilon\left(a H(1-\epsilon)^{2}\right)^{-1}$

and the assumption that the last term on the rhs of Eq.

is small in comparison with the first term.

In terms of $\tau$ Eq. (16) for the Fourier transform $\phi(\mathbf{k})$ reads $(k=|\mathbf{k}|)$

$$
\begin{aligned}
\left(\partial_{\tau}^{2}\right. & -\frac{2+3 \tilde{\Gamma}}{1-\epsilon} \frac{1}{\tau} \partial_{\tau}+k^{2} \\
& \left.+\frac{3 \delta+\frac{9}{2} \tilde{\Gamma}}{(1-\epsilon)^{2}} \tau^{-2}\right) \phi=\gamma \beta^{-\frac{1}{2}} \eta_{\tau} .
\end{aligned}
$$

Let

$\zeta=k \tau$

and

$v^{2}=(1-\epsilon)^{-2}\left(\frac{9}{4}-3 \delta-\frac{3}{2} \epsilon++\frac{9}{4} \tilde{\Gamma}^{2}-\frac{3}{2} \epsilon \tilde{\Gamma}\right)$

(the term $-\frac{3}{2} \epsilon$ in Eq. (39) is replaced by $+\frac{9}{2} \epsilon$ in the corresponding formula in [28] owing to the contribution of gravitational modes as expressed by scalar perturbations).

The calculation of the expectation value of the solution of Eq. (37) over the noise $\eta$ leads to

$\left\langle\phi^{2}\right\rangle \simeq k^{-3} \zeta^{2 \mu} Y_{v}^{2}(\zeta)\left(U^{\prime}\left(\phi_{c}\right)\right)^{2} \simeq k^{-3} \zeta^{2 \mu-2 v}\left(U^{\prime}\left(\phi_{c}\right)\right)^{2}$

for small $k$ (as the Bessel function $Y_{v}(\zeta) \simeq \zeta^{-v}$ for small $\zeta)$.Here

$\mu=(1-\epsilon)^{-1}\left(\frac{3}{2}-\frac{\epsilon}{2}+\frac{3}{2} \tilde{\Gamma}\right)$.

In Eq. (40) the time $\mathrm{t}$ in $\phi_{c}(t)$ must be replaced by $\tau$ then $\tau$ is expressed as $\frac{\zeta}{k}$. For a small $\tilde{\Gamma}$ and $U^{\prime} \simeq$ const we have in a linear approximation in the indices describing the interaction corrections:

$2 v=3+2 \epsilon-2 \delta$,

$2 \mu=3+2 \epsilon+3 \tilde{\Gamma}$.

From Eqs. (40-43) if $U^{\prime} \simeq$ const

$\left\langle\phi^{2}\right\rangle \simeq k^{-3+2 \delta+3 \tilde{\Gamma}}$.
If $U(\phi)=\phi$ and the term $\frac{3}{2} \gamma^{2} H \phi$ is absent in Eq. (16) then in [28] we obtained the power spectrum $k^{-3+2 \eta}$ which agrees with the corresponding result in [19] (in fact, our spectral index $2 \mu-2 v$ in [28] is equal to $2 v-2 \alpha$ of Ref. [19]). For a general $U(\phi)$ our wave equation (16) is different from that of Ref. [19]. The spectral indices in models of warm inflation in $[18,22,23]$ agree with our results when they are concerned with spectral indices of the inflaton. However, there are other fields in those models described by their entropy and densities which additionally contribute to the power spectrum leading to a different spectral index.

\section{The power spectrum of diffusion}

The solution $\phi_{\eta}=\phi_{c}+\phi$ of Eq. (13) with a given initial condition is a sum of the solution $\phi_{c}$ of the homogeneous equation (15) with this initial condition and $\phi$ with 0 as an initial condition at $t_{0}$. From the diffusion approximation (26) we obtain

$$
\begin{gathered}
\rho_{t}(\mathbf{k})=\beta^{-1} \gamma^{2} \int_{t_{0}}^{t} \exp \left(-2 \int_{s}^{t} v\right) \\
a(s)^{-3}\left(3 H+\tilde{\gamma}^{2}\right)^{-2} U^{\prime}\left(\phi_{c}\right)^{2} d s .
\end{gathered}
$$

We can have $\rho_{t} \simeq k^{-2 \kappa}$ with $\kappa>0$ if $t_{0}=-\infty$ (otherwise the integral (45) would be finite at $k=0$ ). For $a=\exp \left(\int_{t_{0}}^{t} H\left(t^{\prime}\right) d t^{\prime}\right)$ this means that the initial condition is at $a\left(t_{0}=-\infty\right)=0$. If we introduce the e-fold time

$d v=H d t$

then

$$
\begin{aligned}
\rho_{t}(\mathbf{k})= & \beta^{-1} \gamma^{2} \\
& \int_{v\left(t_{0}\right)}^{v(t)} \exp \left(-2 \int_{\tau}^{v(t)}(1+\tilde{\Gamma})^{-1}\right. \\
& \left.\left(\delta+\left(3 H^{2}\right)^{-1} \exp \left(-2 \tau^{\prime}\right) k^{2}+\frac{3}{2} \tilde{\Gamma}\right) d \tau^{\prime}\right) \\
& \exp (-3 \tau)\left(U^{\prime}\left(\phi_{c}(\tau)\right)^{2} H^{-1}\left(3 H+\tilde{\gamma}^{2}\right)^{-2} d \tau .\right.
\end{aligned}
$$

(it is assumed that in $\phi_{c}(s)$ the cosmic time has been expressed by the e-fold time). We assume in this section that $\tilde{\Gamma}, \delta, U^{\prime}\left(\phi_{c}\right), H$ and $\left(U^{\prime}\right)^{-2} U^{\prime \prime} U$ are slowly varying in time, so that we may approximate them by a constant.

We introduce the variable

$u=\exp (-2 v)$ 
and assume that $H(v) \simeq$ const then

$$
\begin{aligned}
\rho_{t}(\mathbf{k})= & \frac{1}{2 H} \beta^{-1} \gamma^{2} \exp \left(\left(3 H^{2}\right)^{-1}(1+\tilde{\Gamma})^{-1} k^{2} \exp (-2 v)\right) \\
= & \exp (-2 q v) \int_{u(v)}^{u\left(\nu_{0}\right)}\left(3 H+\tilde{\gamma}^{2}\right)^{-2} U^{\prime}\left(\phi_{c}\right)^{2} \\
& \exp \left(-\left(3 H^{2}\right)^{-1}(1+\tilde{\Gamma})^{-1} k^{2} u\right) u^{\frac{1}{2}-q} d u
\end{aligned}
$$

where

$q=\left(\delta+\frac{3}{2} \tilde{\Gamma}\right)(1+\tilde{\Gamma})^{-1}$

The result of integration in Eq. (47) assuming that $H, U^{\prime}$ $\tilde{\Gamma}$ and $q$ are approximately constant can be expressed by the incomplete $\Gamma$ function

$$
\begin{aligned}
\rho_{t}(\mathbf{k})= & \frac{1}{2 H}\left(3 H+\tilde{\gamma}^{2}\right)^{-2} \exp (-2 q v) \tilde{\gamma}^{2} \beta^{-1} \\
& \exp \left(\left(3 H^{2}\right)^{-1}(1+\tilde{\Gamma})^{-1} k^{2} \exp (-2 v)\right) \\
& \left(\left(\left(3 H^{2}\right)^{-1} k^{2}(1+\tilde{\Gamma})^{-1}\right)^{-\kappa}\right. \\
& \Gamma\left(\kappa,\left(3 H^{2}\right)^{-1}(1+\tilde{\Gamma})^{-1} k^{2} \exp (-2 v)\right) \\
& -\left(\left(3 H^{2}\right)^{-1}(1+\tilde{\Gamma})^{-1} k^{2}\right)^{-\kappa} \\
& \left.\Gamma\left(\kappa,\left(3 H^{2}\right)^{-1}(1+\tilde{\Gamma})^{-1} k^{2} \exp \left(-2 v_{0}\right)\right)\right),
\end{aligned}
$$

where

$\kappa=\frac{3}{2}-q$.

We have for $x<<1$

$\Gamma(\alpha, x)=\Gamma(\alpha)-x^{\alpha} \sum_{n \geq 0}(-x)^{n}(n !(\alpha+n))^{-1}$,

and for $x>>1$

$\Gamma(\alpha, x)=x^{\alpha-1} \exp (-x)$.

If $v_{0} \rightarrow-\infty\left(u\left(v_{0}\right) \rightarrow+\infty\right)$ then the second term in Eq.(51) is vanishing. There remains

$$
\begin{aligned}
\rho_{t}(\mathbf{k})= & \frac{1}{2 H}\left(3 H+\tilde{\gamma}^{2}\right)^{-2} \exp (-2 q v) \tilde{\gamma}^{2} \beta^{-1} \\
& \exp \left(\left(3 H^{2}\right)^{-1}(1+\tilde{\Gamma})^{-1} k^{2} \exp (-2 v)\right) \\
& \Gamma\left(\kappa,\left(3 H^{2}\right)^{-1}(1+\tilde{\Gamma})^{-1} k^{2} \exp (-2 v)\right) \\
& \left(\left(3 H^{2}\right)^{-1} k^{2}(1+\tilde{\Gamma})^{-1}\right)^{-\kappa} .
\end{aligned}
$$

From Eq. (53) only the last term in Eq. (54) is relevant for a small $k$ leading to

$\rho_{t}(\mathbf{k}), \simeq k^{-2 \kappa}$.

where in a linear approximation in the indices $\delta$ and $\tilde{\Gamma}$ we get

$2 \kappa=3-2 \delta-3 \tilde{\Gamma}$

This result agrees with the result (44) obtained from the wave equation in Sect.4. For a large $\Gamma$ Eqs. (40) and (55) with $\kappa$ defined in Eq. (52) also give the same results but power spectrum is far from the scale invariant one in contradistinction to the models in [18] (but in an agreement with the calculations of the spectrum of the inflaton stochastic equation in [19]).

At $\gamma=0$ the result (56) coincides with the power spectrum of quantum fluctuations which are derived by a calculation of $\left\langle\phi^{2}\right\rangle$ in the Bunch-Davis vacuum [8,9](normalized so that the scalar modes behave as plane waves at large $k(a H)^{-1}$; see also a later discussion in [11,30-32] (sec. 24.3)). Our results agree with the results of [19] (also with the calculations of $[18,23]$ when the authors calculate the power spectrum of the stochastic equations). In comparison with [19] one should take into account that we have a different friction term than the authors in[19] and the term $\frac{3}{2} \gamma^{2} H\left(U^{\prime 2}+U U^{\prime \prime}\right)$ is absent in [19]). For $U(\phi)=\phi$ we have calculated the spectrum of the same model as in [19] in [28] (we consider there an extra term of the form $\sigma \frac{3}{2} \gamma^{2} H \phi$ which for $\sigma=0$ corresponds to the case of [19]). Then, our results agree. For general $U(\phi)$ the spectrum of the inflaton equation of [19] cannot be compared with Eq. (16) without additional calculations. It follows from Eq. (54) that the amplitude of thermal fluctuations is determined by $H, \kappa$ (known from CMB measurements [34,35]), $\beta$ and $\gamma$ (which this way would be fixed by $\left.\rho_{t}(\mathbf{k})\right)$. On the other hand the friction $\gamma$ is related (depending on the model) to other measurable quantities as ,e.g., the diffusion constant [36]. In this way the amplitude of stochastic thermal fluctuations depends on many parameters, whereas the virtue of the quantum result consists in the prediction of its $10^{-5}$ magnitude $[5-7,10,11]$ in agreement with observations. The theory shows that under the assumption of almost exponential expansion both the quantum fluctuations and the thermal fluctuations of the inflaton lead to a small deviation from the scale invariant spectral index (this index is crucial for distinguishing various inflation models on the basis of observational data $[34,35])$. The assumption that thermal fluctuations are of quantum origin does not change essentially the results as at high temperatures at the early stage of the universe quantum theory is well approximated by the classical one. Although CMB shows the quantum Planck spectrum (at all wave lengths) the perturbations of the homo- 
geneous solutions at large wave lengths exhibit no quantum effects.

In the next section we show that the potential $U$ describing an interaction of the inflaton with the environment can shift the spectral index. It may be difficult on the basis of a study of the power spectrum to determine whether the deviation from the scale invariant spectrum discovered in WAMP observations comes from quantum or thermal fluctuations. If the initial state of the universe is Gaussian then further evolution of quantum cosmological perturbations proceeds in a squeezed state with a classical evolution as shown in [3739]. In such a case it would be difficult to discover whether the origin of the universe is of quantum nature. The eventual observation of non-Gaussian correlations [40] in CMB could show that a decoherence of quantum superpositions really takes palace.

\section{Beyond the slowly varying corrections}

In the calculations of the spectrum of the stochastic wave equation in Sect. 4 as well as of the spectrum of the diffusion equation in Sect. 5 we assumed that $H, \delta$ and $\tilde{\Gamma}$ vary so slowly that we can approximate them by constants in the calculation of the power spectrum. The slow variation is consistent with the slow roll approximation usually made for inflation. The calculations in Sect. 4 relied heavily on the assumption of the slow variation. For varying potentials we return to the approximation of Sect. 3 of the wave equation by the diffusion equation. The replacement of the wave equation by diffusion equation is legitimate if $3 H+\gamma^{2}\left(U^{\prime}\right)^{2}$ is large. The estimates of the solutions of the diffusion equation based on Eq. (26) are much easier then the study of the corresponding wave equation. We rewrite the formula (45) for the spectrum in the form

$$
\begin{aligned}
\rho_{t}(\mathbf{k})= & =\beta^{-1} \gamma^{2} \\
& \int_{t_{0}}^{t} \exp \left(-2 \int_{s}^{t}\left(3 H\left(t^{\prime}\right)+\tilde{\gamma}^{2}\right)^{-1}\right. \\
& \left(V^{\prime \prime}+a^{-2} k^{2}+\frac{1}{2} \partial_{t}^{\prime} \tilde{\gamma}^{2}+\frac{3}{2} \gamma^{2} H\left(\left(U^{\prime}\right)^{2}+U^{\prime \prime} U\right)\right) \\
& a(s)^{-3}\left(3 H+\gamma^{2} U^{\prime}\left(\phi_{c}(s)\right)^{2}\right)^{-2}\left(U^{\prime}\left(\phi_{c}(s)\right)^{2} d s .\right.
\end{aligned}
$$

In this formula we admit that $V^{\prime \prime}\left(\phi_{c}(t)\right)$ and $U^{\prime}\left(\phi_{c}(t)\right)$ have a substantial variation in time. This property depends on the potentials as well as on $H$. First, we assume that $H$ is (almost) constant. In the damped wave equation the large time behaviour does not depend on the term $\partial_{t}^{2} \phi$. For small time in order to neglect $\partial_{t}^{2} \phi$ we must make the assumptions that $\left(V^{\prime} V^{-1}\right)^{2}$ and $V^{\prime \prime} V^{-1}$ are small and slowly varying in time (this is the slow-roll approximation). Neglecting $\partial_{t}^{2} \phi_{c}$ in Eq. (15) we can represent this equation in an integral form
$\int\left(V^{\prime}\left(\phi_{c}\right)+\frac{3}{2} \gamma^{2} H U U^{\prime}\left(\phi_{c}\right)\right)^{-1}\left(3 H+\gamma^{2} U^{\prime}\left(\phi_{c}\right)^{2}\right) d \phi_{c}=-t$

Equation (15) will have solutions decaying to zero (or to a constant) as from Eq. (58) $\partial_{t} \phi_{c}$ is negative if $V^{\prime}+\frac{3}{2} \gamma^{2} U U^{\prime}$ is positive. If the decay is exponential

$\phi_{c} \simeq \exp (-b t)$

so that

$U^{\prime}\left(\phi_{c}(t)\right) \simeq \exp (-r t)$

then, as follows from the estimates of Sect. 5 after an insertion of (60) in Eq. (57)

$\rho_{t}(k) \simeq k^{-3-2 r+2 \delta+3 \tilde{\Gamma}}$.

Hence, the decay (60) leads to a shift of the spectral index. The behaviour (61) can really happen as we can see assuming that $V^{\prime}$ is negligible and $\gamma^{2}\left(U^{\prime}\right)^{2}>>3 H$. Then, Eq. (58) has the solution

$U\left(\phi_{c}(t)\right)=A \exp \left(-\frac{3 H}{2} t\right)$.

If $U \simeq \phi^{n}$ then $r=\frac{3 H(n-1)}{2 n}$. An exponential decay will be a common behaviour for polynomial $V$ and $U$ in Eq. (58). Let us consider some examples. $V=\frac{m^{2}}{2} \phi^{2}, U=\phi$ gives a linear equation (15) with the decay rate $b=\left(m^{2}+\frac{3}{2} \gamma^{2} H\right)(3 H+$ $\left.\gamma^{2}\right)^{-1}$. Easily calculated integral (58) for $V=\frac{m^{2}}{2} \phi^{2}$ and $U=\frac{1}{2} \phi^{2}$ gives

$b=r=\frac{m^{2}}{3 K}$.

As a next example if $V=\frac{g}{4} \phi^{4}, U=\phi$ then $b=\frac{1}{2} \gamma^{2}$, but $r=0$ (no effect on the power spectrum in Eq. (61)). The decay can be non-exponential as can be seen if $V=\frac{g}{4} \phi^{4}$ and $U=\frac{1}{2} \phi^{2}$ then

$\phi_{c}^{-2}=\phi_{0}^{-2}+\frac{4 g+3 H \gamma^{2}}{6 H} t$.

In such a case $b=r=0$ and the power spectrum is changed only by logarithmic corrections.

As a different class of models let us consider the powerlaw inflation $a=t^{\alpha}, H=\frac{\alpha}{t}$. Consider the potentials

$V(\phi)=\lambda \exp (4 u \phi)$

and

$U(\phi)=\Lambda \exp (u \phi)$. 
Equation (15) has a solution of the form

$\phi=-\frac{1}{2 u} \ln (t)$

if the parameters satisfy the relation

$\alpha=\frac{1}{3} \frac{1-u^{2} \gamma^{2} \Lambda^{2}+8 \lambda u^{2}}{1-u^{2} \gamma^{2} \Lambda^{2}}$.

We require $\alpha>1$, hence $u^{2} \gamma^{2} \Lambda^{2}<1$.

The $s$-integral in Eq. (57) reads (we choose $t_{0}=0$ so that $\left.a\left(t_{0}\right)=0\right)$

$\rho_{t}=K(t, k) \int_{0}^{t} d s s^{-3 \alpha+h+1} \exp \left(-B(2 \alpha-2)^{-1} k^{2} s^{2-2 \alpha}\right)$,

where $K$ is a certain function bounded for a small $k$,

$$
\begin{aligned}
h= & 2\left(3 \alpha+\gamma^{2} \Lambda^{2} u^{2}\right)^{-1}\left(16 \lambda u^{2}-\frac{1}{2} \gamma^{2} \Lambda^{2} u^{2}\right. \\
& \left.+\gamma^{2} \Lambda^{2} u^{2} \frac{1+8 \lambda u^{2}-\gamma^{2} \Lambda^{2} u^{2}}{1-\gamma^{2} \Lambda^{2} u^{2}}\right), \\
B= & 2\left(3 \alpha+\gamma^{2} \Lambda^{2} u^{2}\right)^{-1} .
\end{aligned}
$$

Performing the integral (68) we obtain the power spectrum (55) with

$\kappa=\frac{3 \alpha-h}{2(\alpha-1)}$.

For $h=1$ the result is the same as in the case of a power spectrum of quantum fields [30] in a metric $a \simeq t^{\alpha}$ (this is the almost scale invariant spectrum for a large $\alpha$ ).

\section{Summary and outlook}

The stochastic wave equation can be considered as a phenomenological effective field theory of an inflaton. In general, in addition to the inflaton there will be other fields which contribute to the power spectrum. In our model the stochastic wave equation arises from an average over an infinite set of scalar fields interacting with the inflaton. We have investigated its long wave power spectrum on a basis of some wellcontrolled approximations. We have shown that the scale invariant spectrum is related to the coordinate-independent form of the noise and to the accelerated expansion of the metric. The diffusion approximation derived in this paper allows to study the inflaton power spectrum beyond the assumption of an almost exponential expansion and small variation of the potentials. We considered a potential $U$ describing interaction with an environment which in the case of an almost exponential expansion shifted the spectral index. In an example of a power-law inflation we have obtained a power spectrum close to the scale invariant one in models with exponential potentials. For a small friction and an almost exponential expansion the departure from the scale invariant spectrum is determined by the same formula as the one obtained from quantization of the scalar field on an external expanding space-time. If there is a friction then Hamiltonian quantum mechanics is not well-defined. However, we suppose that the proper formulation as a dissipative Lindblad theory would lead the same formula for the power spectral index. Our stochastic methods suggest that the spectral long wave index cannot distinguish between quantum inflaton fluctuations and classical thermal fluctuations. The time evolution of cosmological perturbations has been studied in $[37,38]$ with the conclusion that if the inflation starts from a Gaussian state then it quickly becomes classical (decoherence without the environment). The CMB spectrum satisfying the Planck law is certainly quantum but we could not see this in the long wave limit. If we admitted non-Gaussian states then a complete decoherence theory based on the Lindbald equation would be needed [39] in order to explain the structure formation and detect when the classical behaviour begins. From the formula for $\rho_{t}(k)$ in this paper we could conclude that $\rho_{t}(k) \simeq k^{-1}$ for large $k$. This is a quantum behaviour of $\left\langle\phi^{2}\right\rangle$. However, for large $k$ the stochastic equation discussed in this paper is not reliable. One should rather study the interaction with an environment at high momenta initiated in [26].

Data Availability Statement This manuscript has associated data in a data repository. [Authors' comment: All data included in this manuscript are available upon request by contacting with the corresponding author (Zbigniew Haba).]

Open Access This article is licensed under a Creative Commons Attribution 4.0 International License, which permits use, sharing, adaptation, distribution and reproduction in any medium or format, as long as you give appropriate credit to the original author(s) and the source, provide a link to the Creative Commons licence, and indicate if changes were made. The images or other third party material in this article are included in the article's Creative Commons licence, unless indicated otherwise in a credit line to the material. If material is not included in the article's Creative Commons licence and your intended use is not permitted by statutory regulation or exceeds the permitted use, you will need to obtain permission directly from the copyright holder. To view a copy of this licence, visit http://creativecomm ons.org/licenses/by/4.0/.

Funded by $\mathrm{SCOAP}^{3}$.

\section{Appendix A: Invariance under a change of coordinates}

We give a simple proof that the stochastic wave equation without friction (friction comes from an interaction of $\phi$ with an environment as in [26], the low momentum approximation is not invariant under change of coordinates) 
$g^{-\frac{1}{2}} \partial_{\mu} g^{\frac{1}{2}} \partial^{\mu} \phi+V^{\prime}=g^{-\frac{1}{4}} \eta$

is invariant under a change of coordinates, where $g=$ $\left|\operatorname{det}\left[g_{\mu \nu}\right]\right|$. Under the change of coordinates $x \rightarrow y$

$$
\begin{aligned}
\left\langle\eta(x) \eta\left(x^{\prime}\right)\right\rangle & =\delta\left(x-x^{\prime}\right)=\delta\left(x(y)-x^{\prime}\left(y^{\prime}\right)\right) \\
& =\delta\left(y-y^{\prime}\right)\left|\frac{\partial x}{\partial y}\right|^{-1}=\left|\frac{\partial x}{\partial y}\right|^{-1}\left\langle\eta(y) \eta\left(y^{\prime}\right)\right\rangle
\end{aligned}
$$

where $\frac{\partial x}{\partial y} \mid$ is the Jacobian. So

$\eta(x)=\left|\frac{\partial x}{\partial y}\right|^{-\frac{1}{2}} \eta(y)$

On the other hand

$g(x)=g(y)\left|\frac{\partial x}{\partial y}\right|^{2}$

Hence, $g^{-\frac{1}{4}} \eta$ is invariant (in a flat expanding metric this is $\left.a^{-\frac{3}{2}} \eta\right)$.

\section{Appendix B: Exact formula for the exponential expansion}

When $a(t)=\exp (H t)$ then the solution of the diffusion can be obtained explicitly. Assume $U(\phi)=\phi$, denote

$M^{2}=V^{\prime \prime}+\frac{3}{2} H \tilde{\gamma}^{2}$

and assume that $M^{2}$ can be approximated by a constant. The solution of the linear diffusion equation (19) with zero initial condition at $t_{0}=-\infty(a(-\infty)=0)$ is

$$
\begin{aligned}
\phi_{t}= & \beta^{-\frac{1}{2}} \tilde{\gamma} \int_{-\infty}^{t} d s \frac{1}{3 H+\tilde{\gamma}^{2}} \eta_{s} \exp \left(-\frac{3}{2} H(t-s)\right) \\
& \exp \left(-\frac{k^{2}}{3 H+\tilde{\gamma}^{2}}(\exp (-2 H s)-\exp (-2 H t))\right. \\
& \left.-\frac{M^{2}}{3 H^{2}+H \tilde{\gamma}^{2}}(t-s)\right)
\end{aligned}
$$

Let

$$
\begin{aligned}
u(s) & =\exp (-2 H s) \\
R & =3 H^{2}\left(1+\frac{1}{3} \tilde{\gamma}^{2} H^{-1}\right)=3 H^{2}(1+\tilde{\Gamma})
\end{aligned}
$$

Then

$$
\begin{aligned}
& \rho_{t}(\mathbf{k})=\frac{\tilde{\gamma}^{2}}{2 H \beta}\left(\frac{1}{3 H+\tilde{\gamma}^{2}}\right)^{2} \exp \left(\frac{k^{2} \exp (-2 H t)}{R}\right) \\
& \int_{u(t)}^{\infty} \exp \left(-\frac{k^{2} u}{R}\right) u^{\kappa-1} d u
\end{aligned}
$$

where

$\kappa=\frac{3}{2}-\frac{M^{2}}{R}$

The integral can be expressed by the incomplete $\Gamma$

$$
\begin{aligned}
& \rho_{t}(\mathbf{k})=\frac{\tilde{\gamma}^{2}}{2 H \beta}\left(\frac{1}{3 H+\tilde{\gamma}^{2}}\right)^{2} \exp \left(\frac{k^{2} \exp (-2 H t)}{R}\right) \\
& \left(\frac{k^{2}}{R}\right)^{-\kappa} \Gamma\left(\kappa, \frac{k^{2}}{R} u(t)\right) .
\end{aligned}
$$

From Eqs.(53) and (80)

$$
\rho_{t}(\mathbf{k}) \simeq k^{-2 \kappa}
$$

If $\gamma=0$ then

$\kappa=\frac{3}{2}-\delta$

with $\delta=\frac{M^{2}}{3 H^{2}}$.

This is exactly the index resulting from a quantization of the scalar field in an exponentially expanding universe [11,30-32].

\section{References}

1. E. Harrison, Phys. Rev. D 1, 2726 (1970)

2. Y.B. Zeldovich, MNRAS 160, 1 (1972)

3. K.T. Story et al., ApJ 779, 86 (2013)

4. P.A.R. Ade et al., arXiv:1502.01589 (2015)

5. A.A. Starobinsky, JETP Lett. 30, 682 (1979)

6. V.F. Mukhanov, G.V. Chibisov, JETP Lett. 33, 532 (1981)

7. A.A. Starobinsky, Phys. Lett. 117B, 175 (1982)

8. A.D. Linde, Phys. Lett. B 116, 335 (1982)

9. A. Vilenkin, L.H. Ford, Phys. Rev. D 26, 1231 (1982)

10. A.H. Guth, S.-Y. Pi, Phys. Rev. Lett. 49, 1110 (1982)

11. A.O. Linde, JETP Lett. 40, 1335 (1984)

12. J. Bardeen, Phys. Rev. D 22, 202 (1980)

13. V.F. Mukhanov, H.A. Feldman, R.H. Brandenberger, Phys. Rep. 215, 203 (1992)

14. A.A. Starobinsky, JETP Lett. 42, 152 (1985)

15. F. Finelli, G. Marozzi, G.P. Vacca, G. Venturi, Phys. Rev. D69, 123508 (2004)

16. F. Finelli, G. Marozzi, A.A. Starobinsky, G. Venturi, Phys. Rev. D 79, 044007 (2009)

17. V. Vennin, A.A. Starobinsky, Eur. Phys. J. C 75, 413 (2015)

18. M.H. Hall, I.G. Moss, A. Berera, Phys. Rev. D 69, 083525 (2004)

19. R.O. Ramos, L.A. da Silva, JCAP 03, 032 (2013) 
20. A. Berera, Nucl. Phys. B 585, 666 (2000)

21. A. Berera, I.G. Moss, R.O. Ramos, Rep. Progr. Phys. 72, 026901 (2009)

22. A.N. Taylor, A. Berera, Phys. Rev. D 62, 083517 (2000)

23. Ch. Graham, I.G. Moss, JCAP7,013 (2009). arXiv:0905.3500

24. A. Berera, Phys. Rev. D 54, 2519 (1996)

25. Z. Haba, Adv. High. Energy. Phys. 2018, 7204952 (2018). arXiv:1807.00639

26. Z. Haba, Eur. Phys. J. C 80, 321 (2020). arXiv:2001.07268(gr-qc)

27. B.A. Bassett, S. Tsujikawa, D. Wands, Rev. Mod. Phys. 78, 537 (2006)

28. Z. Haba, Phys. Lett. B 792, 320 (2019)

29. R.P. Woodard, Int. J. Mod. Phys. D 2014, 231430020 (2014)

30. L.F. Abbott, M.B. Wise, Nucl. Phys. B 244, 541 (1984)

31. F. Takahashi, Wen Yin, A.H. Guth, Phys. Rev. D 98, 015042 (2018)

32. D.H. Lyth, A.R. Liddle, The Primordial Density Perturbation (Cambridge University Press, Cambridge, 2009)
33. K. Bhattacharya, S. Mohanty, R. Rangarajan, Phys. Rev. Lett. 96, 121302 (2006)

34. E.J. Copeland, E.W. Kolb, A.R. Liddle, J.E. Lindsey, Phys. Rev. Lett. 71, 219 (1993)

35. E.J. Copeland, J.E. Lindsey, A.R. Liddle, E.W. Kolb, T. Barreiro, M. Abney, Rev. Mod. Phys. 69, 374 (1997)

36. Z. Haba, A. Stachowski, M. Szydlowski, JCAP 07, 024 (2016)

37. A. Albrecht, P. Ferreira, M. Joyce, T. Prokopec, Phys. Rev. D 50, 4807 (1994)

38. D. Polarski, A.A. Starobinsky, Class. Quantum Gravity 13, 377 (1996)

39. C. Kiefer, D. Polarski, Adv. Sci. Lett. 2, 164 (2009). arXiv:0810.0087

40. J. Maldacena, JHEP 05, 013 (2003) 